\title{
Microbial growth and metabolism: Modelling and calorimetric characterization
}

\author{
Alberto Schiraldi \\ DISTAM, University of Milan, Via Celoria 2, 20133 Milano, Italy
}

\begin{abstract}
Microbial growth can be qualitatively described with the empirical Gompertz function. This, however, has no specific physical meaning and gives no information about the underlying biochemical activity. When used to fit the isothermal calorimetric traces obtained from microbial cultures, it is inadequate. A more satisfactory description comes from a kinetic model that can reproduce the plate count data, the isothermal calorimetric trace and the change in the metabolite concentration.
\end{abstract}

\section{INTRODUCTION}

Predictive microbiology has recently attracted increasing interest because of its direct use to assess food safety. The main target of these studies is the growth of the microbial population in a given environment, so as to reproduce the experimental plate counts of the Colony Forming Units (CFU). When plotted is time, the plate count data show a sigmoidal trend and therefore can be satisfactorily fitted by any function that can reproduce such a behaviour. One of these, namely the Gompertz function, $G(t)$, was found (1) more adequate than others to work out the experimental findings:

$$
G(t)=\ln \frac{\mathrm{N}}{\mathrm{N}_{0}}=a \times \exp [-\exp (b-c \times t)]
$$

It accounts for the conventional parameters describing the microbial growth in isothermal conditions (see Fig. 1): namely, microbial population, $N$, lag-phase, $\lambda=(h-1) / c$, wich is the time span preceding the growth onset, plateau level, $\ln \left(\mathrm{N}_{\max } \mathrm{N}_{\alpha}\right)=a$ (where $\mathrm{N}_{\mathrm{o}}$ is the starting microbial population), and maximum growth rate, $\mu=a \cdot c / e$ ( $e$ being the base of natural logarithms). The inadequacy of the Gompertz function has recently been the subject of specific works (2-4) and can be summarized as follows

1. $(i(t=0) \neq 0$;

2. $G(t)$ actually is a one-parameter function, since $a, b$ and $c$ are mutually correlated (3),

3. $G(t)$ does not correspond to any specific model of the microbial metabolism: it neither gives details about the lag-phase, nor accounts for microbial death

$G(t)$ was indeed found adequate to fit the plate count data from the cultures of some microbial species, namely, Saccharomyces cerevisiae (5), Lactobacillus hulgaricus and Strepococcus thermophilus (6). It is easy to recognize (see Fig. 2) that the first derivative of $\exp [G(t)]$, viz., the microbial growth rate, is a peak-shaped function that seems a suitable candidate to fit the isothermal calorimetric trace obtained from a growing microbial culture. However, when tentatively applied to fit those from these three cultures, it provided statistically reliable results only for the last two species, and was rather inadequate for the first. As an alternative approach, a kinetic model was proposed to naively mimic the main methabolic processes within the microbial culture and accordingly reproduce both the observed sigmoidal increase of cell population and the relevant isothermal calorimetric trace.

\section{MATERIALS AND METHODS}

In a series of investigations reported in other works (5-7) about fundamental microbiological processes in food industry, such as alcohol fermentation ( $S$. cerevisiae), yogurt (L. bulgaricus, S. thermophilus), and vinegar (Acetobacter aceti) production, isothermal calorimetric traces were recorded after microbial inoculation of a suitable cultural broth Either yeasts or bacteria were investigated by means of a SETARAM C80D twin calorimeter equipped with special cells that allow direct inoculation of a microbial culture into the broth taken at constant temperature. Other details are given in the works mentioned above (5-7) 


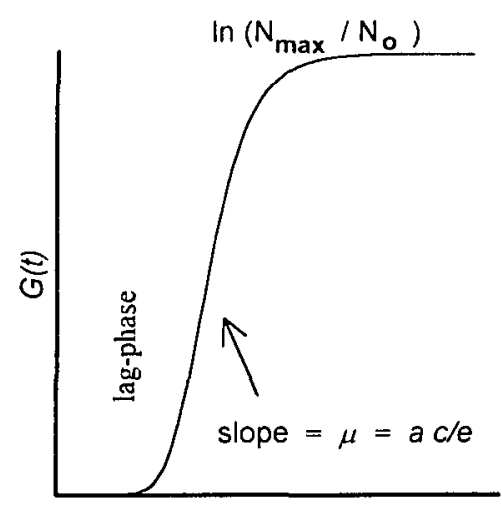

time

Fig. 1 Microbial growth according to the Gompertz function, $G(t)$. The parameters mentioned in the leoends are those reported in the text.

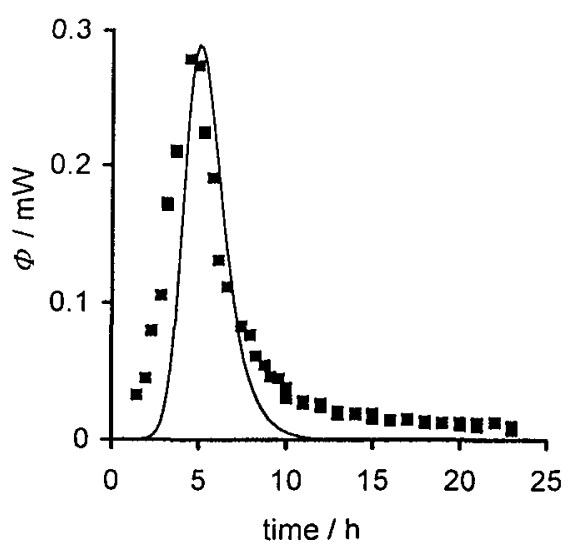

Fig. 2 Calorimetric signal from Streptococcus thermophilus culture, $\mathbf{a}$, and derivative of $\exp [G(t)]$ (arbitrary units),

The traces selected for the present work were subjected to a mathematical treatment that required a previous conversion of the instrument output into ASCII files; these were treated according to special calculation routines implemented in dedicated softwares, namely TableCurve and PeakFit (Jandel, Germany). Another software, namely PC-MATLAB (MathWorks, Inc. USA), was used to numerically integrate systems of differential equations related to a kinetic model of the microbial growth and anabolism. Ethanol and glucose concentration data are those reported in another work from our laboratory $(5)$

\section{RESULTS AND DISCUSSION}

When $G(t)$ is employed to fit an isothermal calorimetric trace from a microbial culture, a naive model can be used that implies two main contributions, namely from the cell duplication, $d Q_{g r} / d /$, and from the metabolic activity, $\mathrm{d} Q_{m e t} / \mathrm{d} t$ (not directly related to the former process), thus proportional to $\mathrm{dN} / \mathrm{d} t$ and to $N$, respectively.

(i) $\mathrm{d} Q_{z_{r}} / \mathrm{d} t$,

$$
\frac{\mathrm{d} Q}{\mathrm{~d} t}=\frac{\mathrm{dN}}{\mathrm{d} t} \times q_{g r}
$$

(ii) $\mathrm{d} Q_{\text {met }} / \mathrm{d} t$,

$$
\frac{\mathrm{d} Q_{m e t}}{\mathrm{~d} t}=\mathrm{N} \times \frac{\mathrm{d} q_{m e t}}{\mathrm{~d} t}
$$

where $q_{g r}$ and $q_{\text {met }}$ stand for heat amount per cell. Accordingly, the overall heat flow rate would be:

$$
=\left(\mathrm{N} \times \frac{\mathrm{d} q_{m e t}}{\mathrm{~d} t}\right)+\left(\frac{\mathrm{dN}}{\mathrm{d} t} \times q_{g r}\right)=\mathrm{N}_{0} \times \exp [G(t)] \times\left[\frac{\mathrm{d} q_{m e t}}{\mathrm{~d} t}+q_{g r} \times \frac{\mathrm{d} G(t)}{\mathrm{d} t}\right]
$$

Once the experimental calorimetric trace is converted into an ASCII file, it can be fitted according to eqn. (4), $q_{g^{r}}$ and $\mathrm{d} q_{\text {met }} / \mathrm{d} t$ being treated as constant quantities when an excess feeding substrate is present. This approach could be easily extended to traces obtained with isoperibolic calorimeters ( 8 ).

Isothermal DSC traces related to the lactic fermentation sustained by $L$. bulgaricus and $S$. thermophilus were satisfactorily reproduced through this procedure (6): Fig. 3 shows the case of a culture of $L$. bulgaricus. The agreement found was indeed rather fortuitous, since in other cases, such as alcoholic fermentation and vinegar production sustained by S. cerevisiae and Acetohacter acefi (6) respectively, and growth of spore-forming Bacillus cereus $(9,10)$, this approach clearly failed. Another argument ayainst 

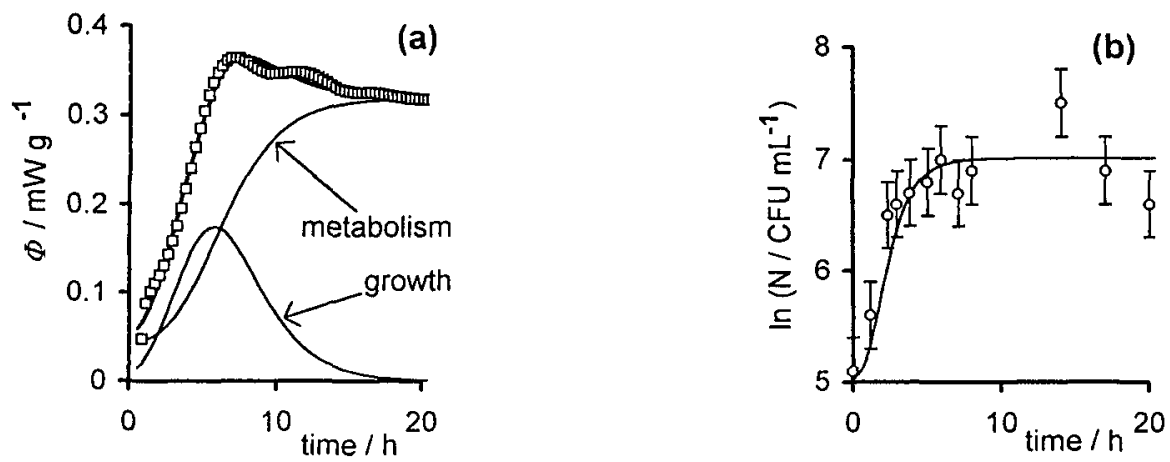

Fig. 3 Culture of L. bulgaricus at $35^{\circ} \mathrm{C}$ in excess cow's milk. The Gompertz function allows reproduction of: (a) the calorimetric trace according to eqn. 4 (referred to the unit mass of culture broth), and (b) the trend of plate count experimental data (O). The former is the sum (i) of two contributions, namely growth (peak shaped) and metabolism (sigmoidal curve)

this procedure comes from its purely phenomenological character: no insight is gained about the metabolic activity. For example, the so-called lag-phase is actually treated as a dormant state, whereas it should correspond to a significant microbial activity, inasmuch as the inoculated culture is adjusting its metabolism to the new environment (3).

Significant improvements can be obtained with a suitable kinetic model that reproduces the sigmoidal increase of the microbial population as a result of specific metabolic steps, which are responsible for the thermal effects observed.

As a general consideration, it should be noted that routine plate counts do not distinguish resting from ready-to-duplicate cells: either cell type is accordingly referred to as a CFU. The method cannot therefore give information about the corresponding heat release. To overcome the mismatch between plate count and calorimetric findings, a scheme of consecutive steps can be proposed, so as to account for both the sigmoidal growth curve and the metabolic activity that prepares the oncoming cell duplication (cell death is here disregarded for the sake of simplicity):

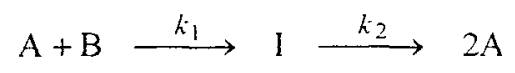

where A stands for a cell that consumes the substrate B to change into the reproductive state $I$. At any time the overall cell population is $N=\left(c_{A}+c_{I}\right)$. The system of differential equations related to the above scheme, viz.,

$$
\begin{aligned}
& \frac{\mathrm{d} c_{\mathrm{A}}}{\mathrm{d} t}=-k_{1} c_{\mathrm{A}} c_{\mathrm{B}}+2 k_{2} c_{\mathrm{I}} \\
& \frac{\mathrm{d} c_{\mathrm{B}}}{\mathrm{d} t}=k_{1} c_{\mathrm{A}} c_{\mathrm{B}} \\
& \frac{\mathrm{d} c_{\mathrm{I}}}{\mathrm{d} t}=k_{1} c_{\mathrm{A}} c_{\mathrm{B}}-k_{2} c_{\mathrm{I}}
\end{aligned}
$$

can be numerically integrated according to the Runge Kutta method. Here $c_{\mathrm{A}}$, $c_{\mathrm{B}}$, and $c_{\mathrm{I}}$ stand for the respective concentrations. Since $A$ and I stand for cell types, while B stands for substrate, i.e. some chemical compound, the relevant concentration units were chosen so as to face comparable figures: $10^{4}$ CFU mL $\mathrm{mL}^{-1}$ (colony forming units, i.e. living cells, per milliliter) for $c_{\mathrm{A}}$ and $c_{\mathrm{I}}$, and $\mathrm{U} \mathrm{mL}^{-1}$, i.e. the stoichiometric substrate equivalent of $10^{4} \mathrm{CFU}$, respectively. The physical units for the kinetic constants can be accordigly assessed. The introduction of the intermediate state, I, which precedes cell duplication, also implies the appearance of a phenomenolgical lag-phase in the microbial growth, as a result of an early decrease of the starting cell population that is soon counterbalanced by new cell generation (see Fig. 4). Accordingly, the lag-phase is not described as a dormant state, but as the transition between two different states. Once all the available substrate has been consumed, the cell population attains a plateau level represented by non-proliferative A cells. 


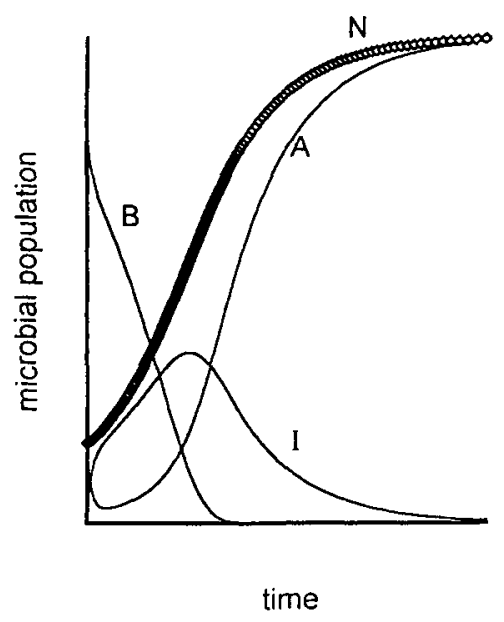

Fig.4 Trend of microbial population, $\mathrm{N}, c_{\mathrm{A}}$ and $c_{I}$, and substrate concentration, $c_{B}$ : the lag-phase is the result of a balance between $A$ and I cells.

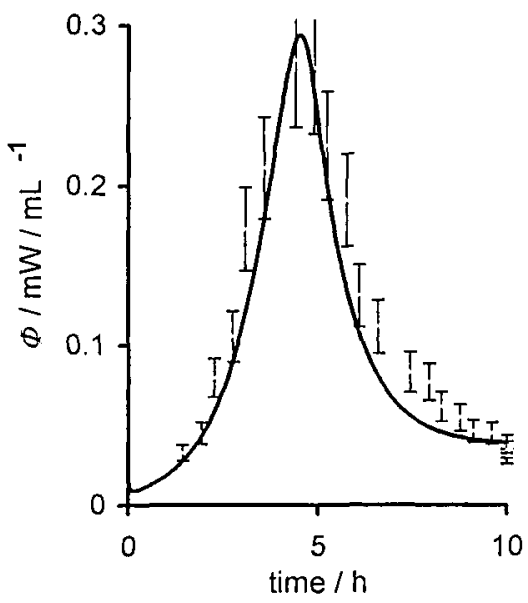

Fig.5 S. thermophilus: experimental and predicted isothermal calorimetric trace. The heat flow rate is referred to the unit volume of culture broth.

The contribution of the cell growth to the overall power release is now described as:

$\frac{\mathrm{d} Q}{\mathrm{~d} t}=\Delta_{1} H\left(k_{1} c_{\mathrm{A}} c_{\mathrm{B}}\right)+\Delta_{2} H\left(k_{2} c_{\mathrm{I}}\right)$

where the enthalpy changes $\Delta_{1} H$ and $\Delta_{2} H$ concern the two kinetic steps. The resulting calorimetric signal would accordingly have a bell or peak shape with a tail that reaches the abscissa: This only occurred in a few cases. More frequently the main signal attains a horizontal trend above or below zero

To explain this finding, it is expedient to remember that even resting cells must spend energy. The relevant overall work is proportional (11) to $N$ and can be described by adding a further step to the kinetic model, namely,

$$
\mathrm{A}\left(\text { and I) } \stackrel{k_{3}}{\longrightarrow} \mathrm{A}\right. \text { (and I) }
$$

with an associated exothermic effect $\Delta_{3} H$ so that,

$$
\stackrel{\mathrm{d} Q_{m \cdot t}}{\mathrm{~d} \ell}=\Delta_{3} H\left(K, c_{\Lambda}\right)
$$

The trace (see Fig. 5) was then reproduced with adequate $\Delta_{1} H, \Delta_{2} H$, and $\Delta_{3} H$ values

A further step can be added to account for the cell death with production of catabolites and an endothermic effect, $\Delta_{4} H$.

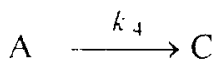

The overall trace would therefore be reproduced by the expression

$\Phi=\left[k_{1} c_{\mathrm{A}} c_{\mathrm{B}} \Delta_{1} H+k_{2} c_{1} \Delta_{2} H\right]+\left[k_{3} \mathrm{~N} \Delta_{3} H\right]+\left[k_{4} c_{\mathrm{A}} \Delta_{4} H\right]$

The energy balance is therefore explained with an a priori scheme that implies separate contribution, exo or endothermic, each from an isothermal kinetic step. 
When applied to $S$. thermophilus (see Fig. 5), a reasonable agreement with the experimental trace was found with the following values

$c_{1}(\mathrm{t}=0)=10^{4} \mathrm{CFU} \mathrm{mL}^{-1}$;

$c_{13}=100 \mathrm{U} \mathrm{mL}^{-1}\left(\mathrm{U}\right.$ being the substrate uptake per $10^{4} \mathrm{CFU}$; see above);

$k_{1}=0.2 \mathrm{~h}^{-1} \mathrm{U}^{-1} \mathrm{~mL}$,

$k_{2}=1 h^{-1}$

$k_{:}=1 \mathrm{~h}^{-1} 10^{-4} \mathrm{CFU}^{-1} \mathrm{~mL}$;

$\Delta_{1} H, \Delta_{2} H$, and $\Delta_{3} H, 27,24.3$, and $1.35 \mathrm{~mJ} 10^{-4} \mathrm{CFU}^{-1}$; respectively.

It must be noted that, according to this model, the maximum of the calorimetric peak occurs just before the flexus of the overall growth curve. Support for this point comes from the observation that the increase of the intracellular ATP precedes that of the microbial population (see Fig. 6): this was observed for cultures of $S$ cerevisiac (5) and B. cereus $(9,10)$.

It is indeed reasonable to assume that ready-to-duplicate cells should be provided with large ATP amounts: accordingly the first step of the kinetic model would correspond to the production of anabolites (including DNA and / or RNA) that prepare cell duplication.

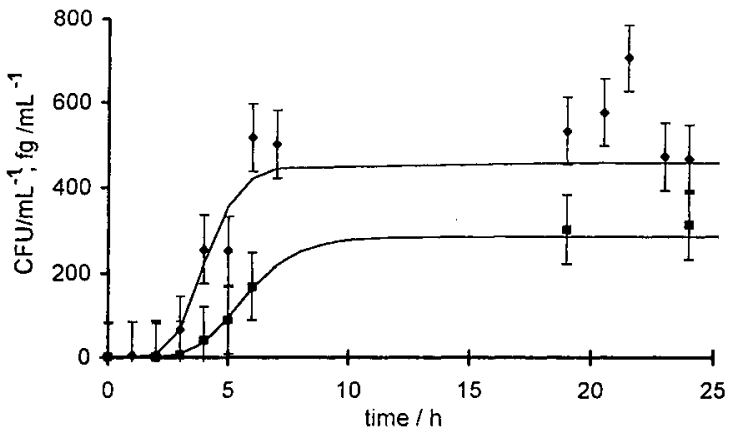

Fig. 6 Increase of microbial population, $\boldsymbol{a}$, and intracellular ATP, $\diamond$, in a culture of Bacillus cereus at $30^{\circ} \mathrm{C}$. ATP is given in femtograms $\mathrm{mL}^{-1}$

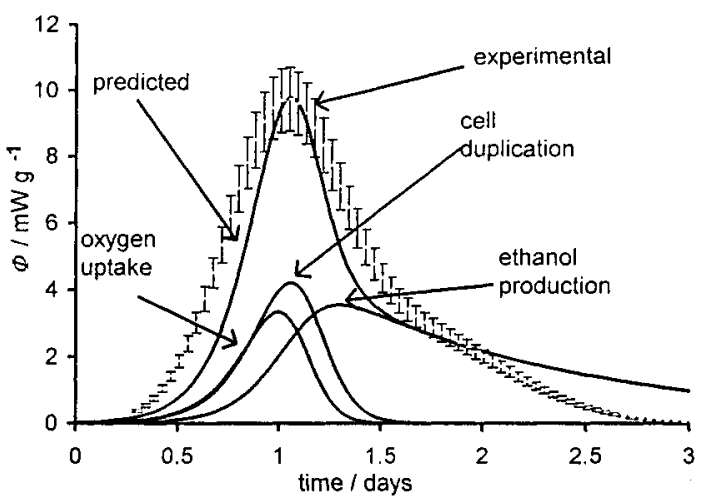

Fig.7 Isothermal calorimetric trace from $S$. cerevisice culture at $35^{\circ} \mathrm{C}$. The underlying peaks refer to the steps defined according to the kinetic model in the text The calorimetric signal, $\Phi$, is referred to the glucose mass

Another example is offered by the alcoholic fermentation sustained by S. cerevisiae

In a previous work (7) traces obtained from this kind of cultures were naively deconvoluted into a sum of gaussian functions just to account for an early aerobic process, where yeast cells undergo duplication, followed by the real anaerobic fermentation process that results in the production of ethanol. Although this treatment of the experimental data allowed the respective reaction yields to be reproduced, no correlation was proposed with the plate count results. With the Gompertz function an improvement was obtained (5), though only in a phenomenological sense. A better description was eventually reached by adjusting the kinetic model to account for the anaerobic production of ethyl alcohol and for cell duplication in the early aerobic conditions.

$$
\begin{aligned}
& \mathrm{A}+\mathrm{O} \stackrel{k_{1}}{\longrightarrow} \mathrm{I} \\
& \mathrm{I} \stackrel{k_{2}}{\longrightarrow} 2 \mathrm{~A} \\
& \mathrm{~A}+\mathrm{G} \stackrel{k_{3}}{\longrightarrow} \mathrm{E}+\mathrm{A}
\end{aligned}
$$

where $O, G$ and $E$ stand for oxygen and glucose uptake, and ethanol production per CFU

The amount of available oxygen directly affects the shape of the trace. When little oxygen is availaible, the calorimetric signal is like that reported in Fig. 7, which came from a culture with starting concentrations, $c_{(;, 0)}=40 \mathrm{~g} \mathrm{~L}^{-1}$ and $c_{\mathrm{A}, 0}=10^{2} \mathrm{CFU} \mathrm{mL}$.

The oxygen amount was expressed in $\mathrm{U}_{0}$ units, $\mathrm{U}_{0}$ being the oxygen uptake per $10^{2} \mathrm{CFU}$; with this choice the starting oxygen concentration which allowed the best fitting was $10^{3} \mathrm{U}_{0} \mathrm{~mL}^{-1}$. The final cell population was $10^{5} \mathrm{CFU} \mathrm{\textrm {mL } ^ { - 1 }}$ 


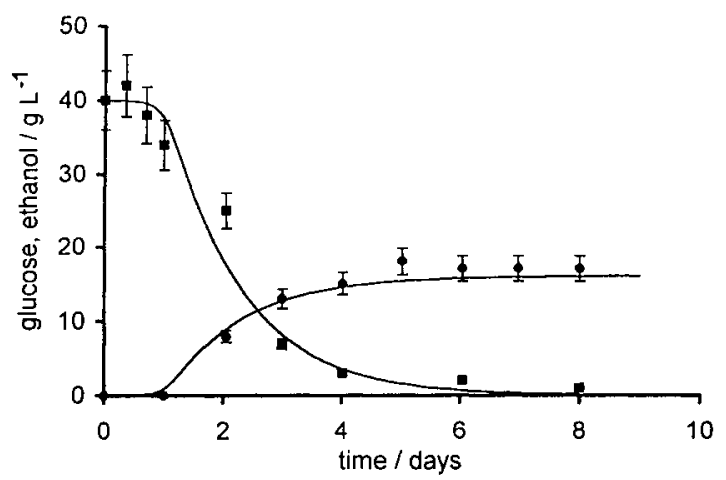

Fig. 8 Glucose, $\mathbf{m}$, and ethanol, $\bullet$, concentration in the course of Saccharomyces cerevisiae fermentation: the fitting curve comes from the kinetic model in the text.

The following parameters gave the best fit:

$k_{1}=310^{-2} \mathrm{~h}^{-1} \mathrm{U}_{\mathrm{O}}^{-1}\left(c_{\mathrm{A}, 0}\right)^{-1}, k_{2}=10 \mathrm{~h}^{-1}, \mathrm{k}_{3}=810^{-4} \mathrm{~h}^{-1} \mathrm{~g}^{-1} \mathrm{~L}\left(c_{\mathrm{A}, 0}\right)^{-1}$,

$\Delta_{1} H=5.4 \mathrm{~mJ} \mathrm{~g}^{-1}, \Delta_{2} H=7.2 \mathrm{~mJ} \mathrm{~g}^{-1}$, and $\Delta_{3} H=555 \mathrm{~J} \mathrm{~g}^{-1}$ (thus expressed per gram of glucose).

The Pasteur stoichiometric yield implies $E / G$ (molar ratio) $=2$; accordingly one should assume that $\mathrm{dE} / \mathrm{d} t=0.5\left(k_{3} c_{\mathrm{A}} c_{\mathrm{G}}\right)$, if $\mathrm{c}_{\mathrm{G}}$ is given in $\mathrm{g} \mathrm{L}^{-1}$. The best fit of the experimental data (see Fig. 8) was however obtained with $\mathrm{dE} / \mathrm{d} t=0.4\left(k_{3} c_{\mathrm{A}} c_{\mathrm{G}}\right)$, which implies $12 \%$ glucose consumed in some other way, such as production of glycerol, succinate, and acetate $(12,13)$. This substrate/product yield, has been reported (12) to be accompanied by a thermal effect of $542 \mathrm{~J} \mathrm{~g}^{-1}$ (calculated from the original data of heat yield and biomass/substrate yield) which is rather close to the $\Delta_{i} H$ obtained in the present study.

The scheme could be easily adjusted to include a death rate affected by the concentration of some specific catabolite, such as ethanol: for the sake of simplicity this was not considered in the present work so as to limit the simulation to 8 parameters, namely the three kinetic constants and related enthalpy changes, and glucose and oxygen concentration

\section{CONCLUSIONS}

A kinetic model with one or more intemediate steps can account for the metabolic activity that precedes cell duplication and reproduce both the sigmoidal growth curve that fits plate count data and the isothermal calorimetric traces obtained from microbial cultures. The model applies to the use of microbial cultures in food productions and cannot be directly compared with the stoichiometric models (11-13) of mass and energy balance dealing with the control of the exponential phase of biomass growth. Our approach indeed seems more adequate to describe the evolution of microbial cultures and therefore more reliable in predictive microbiology studies.

\section{REFERENCES}

1. Zwietering, J.T. De Koos, B.E. Hasenack, J.C. De Witt, and K. van't Riet, App. Environ. Microbiol., 57,1094 (1991).

2. A.M. Gibson, N. Bratchell, and T.A. Roberts, Int. J. Food Microbiol., 6, 155 (1988).

3. J. Baranyi, T.A. Roberts, and P. Mc Clure, Food Microbiol., 10, 43 (1993) and IMA J. Mathem. Appl. Medicine and Biology, 10, 293 (1993).

4. J.P. Sutherland, and A.J. Bayliss, Int. J. Food Microbiol., 21, 197 (1994).

5. Franzetti, E. Mariani, M. Riva, and A. Galli, Ann. Microbiol. Enzimol, 44, 237 (1994).

6. L. A Galli, E.Mariani, L.Franzetti, M.Riva, and A.Schiraldi, Proc. Gth ESTAC (Grado, Italy), p. 106 (1994)

7. A.Schiraldi and M. Riva, Trends in Ecological Physical (hemistry, p. 167, Elsevier, Amsterdam, London, New York, Tokyo (1993).

8. P. Weppen, Thesis (Oldenburg, 1986)

9. G. Praderio, A. Schiraldi, C. Sorlini, A. Stassi, and E. Zanardini, Thermochim. Acta, 227, 205 (1993)

10. A. Schiraldi, E Zanardini, D Daffonchio, and C. Sorlini, Proc. Gih ESTAC (Grado, Italy), p. 272 (1994).

11. B. Birou, U. von Stockar, Linzyme Microh. Technol., 11, 12 (1989)

12. L. C. M. Auberson, and U von Stockar, Biolech. Bioeng, 40, 1243 (1992).

13. C. Larsson, A. Blomberg, and L. Gustafsson, Biotech. Bioeng, 38, 447 (1991). 